\title{
Optimal Placement and Sizing of DG for Loss Reduction,Voltage Profile Improvement and Voltage Sag Mitigation
}

\author{
S. M. Farashbashi-Astaneh ${ }^{1,2}$, A. Dastfan ${ }^{1}$ \\ ${ }^{1}$ Department of Electrical and Robotic Engineering \\ Shahrood University of Technology, Shahrood, Iran \\ E-mail: m.farashbashi@gmail.com,dastfan@ieee.org \\ ${ }^{2}$ Khorasan Regional Electric Company, Mashad, Iran
}

\begin{abstract}
Increasing trend in using distributed generation has motivated power sector planners to consider approaches that result in benefiting the most from investments in this field. Meanwhile subjects such as power loss reduction, voltage profile improvement and voltage sag mitigation has significant role in lessoning imposed expenditures to utility companies. Loss reduction is important for utility companies as it directly increases company benefit in a competitive electricity market, beside reaching power quality standards is too important as it has vital effect on customer orientation. In this paper an optimization algorithm is developed to gain these goals all together. In order to evaluate sag mitigation capability of the proposed algorithm, voltage in voltage sensitive buses is investigated. An existing $20 \mathrm{KV}$ network has been chosen as test network. Results show the efficiency of the proposed algorithm in reducing power loss, improving voltage profile and mitigating voltage sags in voltage sensitive buses.
\end{abstract}

\section{Key words}

power loss, voltage profile, voltage sag mitigation, sensitive loads

\section{Introduction}

In a general concept DG (Distributed Generation) is power generation near consumption. Reducing distance between generation centers and consumption colonies has numerous. Literature overview on this subject shows that using DG units in a feeder reduces power loss significantly, even if DG is not supplying a large percentage of the load. The percentage of loss reduction can reach $85 \%$ in different load patterns [1].

On the other hand current flow limitation in long branches lessons the need for reactive power and thus voltage profile is improved. This characteristic, especially in long feeders which suffer from poor voltage profile is an important motivation to benefit DG. Beside growing use of voltage sensitive equipments, growing awareness about "undistorted power in $P C C^{1 "}$ and need for uninterruptible and high quality power to survive in competitive economic markets for commercial and industrial consumers are just a few reasons for the importance of power quality. Considering these facts and high DG penetration level in future grids, using all potential capacities for gaining the most from DG technologies seem evitable. Considering the fact that conventional distribution grids are commonly fed unidirectional, a fault or starting a large size motor in one bus of the grid can cause voltage sag in buses in vicinity [2]. DG supports voltage in connection point [3] and this efficiency is highly dependant on the size and location of DG unit, so optimal sizing and location of DG can give the opportunity to benefit this potential capacity to mitigate voltage sags, particularly in buses with sensitive loads.

In literature valuable studies have been done on assessment the impact of using DG on the power quality of distribution networks. In [4] different sag related indices have been developed and impact of DG penetration levels has been investigated on defined indices. However it should be noted that in network expansion programming, other network operation concerns such as loss minimization and improving voltage profile in different loadings are also important factors. In this study a comprehensive approach is followed.

\section{Genetic Algorithm}

Genetic algorithm is a computational model that relies on concepts of evolutionary processes [5]. The genetic algorithms can be applied in optimization fields. $\mathrm{GA}^{2}$ operates on a population of possible answers (individuals or chromosomes), applying selection and reproduction criteria and by then new solutions (offspring) are generated containing information from

\footnotetext{
1 - Point of Common Coupling

2 - Genetic Algorithm
} 
where they were originally created (parents).Obviously, the better the solution, there is a higher probability one goes to the next offspring.

The first step is encoding a potential solution in a data structure type. Once the initial population is randomly generated every solution is evaluated by means of a goal (or objective) function. In each generation the fitness value of all individuals are calculated and those with higher fitness values have more probability to be parents of next generation. At this point, some individuals are coupled by means of a crossover operator.

In continue the individuals can undergo mutation, which involves selecting a gene in a string and change it to another possible value. The probability of mutation to happen is rather small.

\section{Problem Formulation}

In this section objective functions that GA uses for optimal sizing and placement for DG units is proposed. Each of these goal functions can be used separately to follow related goal.

\section{A. Power Loss Reduction}

The total active power loss $\left(P_{\text {Loss }}\right)$ in a distribution grid can be calculated as [6]:

$$
P_{\text {Loss }}=\sum_{j=1}^{N-1} I_{j}^{2} R_{j}
$$

Where $I_{j}$ is the magnitude of current for feeder section $\mathrm{j}$. $R_{j}$ is the resistance of feeder $\mathrm{j}$ and $\mathrm{N}-1$ is the total number of feeder sections, in case there are $\mathrm{N}$ nodes in the studied grid. Loss reduction can be achieved by following fitness function:

$$
f_{\text {Loss }}=\frac{1}{P_{\text {Loss }}}
$$

\section{B. Voltage Profile Improvement}

Voltage profile improvement fitness function can be explained as (3).

$$
f_{v}=\frac{1}{N \sqrt{\sum_{j=1}^{j=N}\left(V_{n}-V_{j}\right)^{2}}}
$$

In which $V_{n}$ is voltage level, $V_{j}$ is voltage of the $j^{\text {th }}$ bus and $\mathrm{N}$ is the number of buses of the network under study.

\section{Voltage Support in Voltage Sensitive Buses}

There are two different approaches to define an objective function to maximize voltage support in buses that feed voltage sensitive loads. In the first approach the focus is on the magnitude of voltage in sensitive buses during a voltage sag in eventful buses (buses with a high sag frequency index). With this approach the related objective function can be defined as below:

$$
f_{\text {Sensitive }}=\frac{1}{\sum_{i=1}^{N} K_{i}\left(V_{n}-V_{i, \text { Sensitive }}\right)}
$$

In which $V_{n}$ is voltage level of network, $V_{i, \text { sensitive }}$ is the voltage of $i^{\text {th }}$ sensitive bus in the studied network and $K_{i}$ is the weighting coefficient for the $i^{\text {th }}$ voltage sensitive bus and is defined as below:

For $0.1^{P U} \leq V_{\text {Sensitive }} \leq 0.4^{P U} \quad \cdot \mathrm{K}=0.7$
For $0.4^{P U}<V_{\text {Sensitive }} \leq 0.7^{P U} \quad \cdot \mathrm{K}=0.2$
For $0.7^{P U}<V_{\text {Sensitive }} \leq 0.9^{P U} \quad \cdot \mathrm{K}=0.1$

It means that in a sensitive bus, higher voltage amplitude (during voltage sags in eventful buses) has a higher fitness value and thus proposed algorithm tends to introduce place and size of DG, which lead to this voltage condition. The main hint for choosing pre said limitation for voltage of sensitive buses $\left(V_{\text {Sensitive }}\right)$ is given from IEC 61000-4-11 which recommends levels of $10 \%, 40 \%$ and $70 \%$ as the magnitude values for testing equipment against voltage sags and short interruptions. In [7] this criterion is used to form a objective function for sag mitigation by Flexible AC Transmission System (FACTS) devices.

The second approach deals with voltage sag propagation mitigation. This takes into account the number of buses that experience voltage sag. (5) Shows the proposed function to cover this concept.

$$
f_{S P}=\omega_{1} V_{L}+\omega_{2} V_{M}+\omega_{3} V_{H}(5)
$$

In which $V_{L}$ is the number of buses with voltage amplitude drop below 0.1 p.u, $V_{M}$ is the number of buses with voltage amplitude between 0.4 p.u and 0.7 p.u and finally $V_{H}$ is the number of buses with voltage amplitude between 0.7 p.u and 0.9 p.u during voltage sag occurrence. Weighting coefficients are defined as

$$
\omega_{1}=0.1, \omega_{2}=0.3 \text { and } \omega_{3}=0.6 \text { to magnify }
$$
voltage support in more ill-conditioned buses. In this paper the first approach is used in simulations.

\section{Illustrative Example}

\section{A. Test Network}

An existing $20 \mathrm{KV}$ network which is modeled with 32 buses is studied. This medium voltage feeder which is located in south Khorasan province in Iran has severe voltage problems especially in peak load hours and in end feeder areas. Figure 2 shows single line diagram of this network.

In summer the voltage drops to 0.85 per unit in peak load hours. Utility company in the region has tried to solve the problem with using capacitors but the problem still exists. 


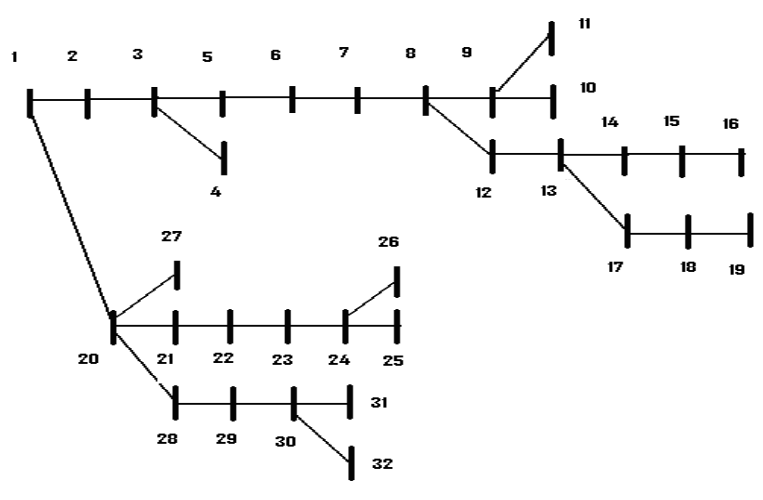

Fig. 1 Single Line Diagram of the Test Network

\section{B. $Y_{\text {BUS }}$ Based Load Flow for Distribution Grids}

Due to high $R / X$ ratio of the sample distribution network which is the result of being long and old, Newton-Raphson and FDLF ${ }^{3}$ load flow algorithms do not lead to reliable answers. On the other hand in proposed algorithm GA calls load flow program several times. Therefore using usual load flow algorithms makes the overall proposed algorithm massive and runtime increases immethodical.On the other hand for modeling voltage sag we need impedance to be connected from eventful bus to ground. So we have to use a $Y_{B U S}$ based load flow algorithm. In this study proposed algorithm in [8] is used. This admittance based load flow program gives precise answers in minimum runtime. Assuming that bus 1 as slack, in bus i we have [7]:

$$
V_{i}^{*} \sum_{i=1}^{N} Y_{i j} V_{j}=P_{i}^{s p}-j Q_{i}^{s p}, i=1,2, \ldots, N, i \neq s(6)
$$

Where s denotes the slack bus

With an appropriate solution guess

$V_{0}=\left[V_{10}, V_{20}, \ldots, V_{N 0}\right]$

The above equation can be written as

$$
\left(V_{i 0}^{*}+\Delta V_{i}^{*}\right) \sum_{j=1}^{N} Y_{i j}\left(V_{j 0}+\Delta V_{j}\right)=P_{i}^{s p}-j Q_{i}^{s p}
$$

By neglecting the second order terms, the following liberalized equation is obtained

$$
\begin{aligned}
& V_{i 0}^{*} \sum_{j=1}^{N} Y_{i j} \Delta V_{j}+\left(\sum_{j=1}^{N} Y_{i j} \Delta V_{j}\right) \Delta V_{i}^{*} \cong \\
& \left(P_{i}^{s p}-Q_{i}^{s p}\right)-V_{i 0}^{*} \sum_{j=1}^{N} Y_{i j} \Delta V_{j}
\end{aligned}
$$

In the first sigma on the left $\mathrm{j}$ can not be qual to $\mathrm{s}$. From Equation (6), we can obtain the following approximated relation.

$$
\sum_{j=1}^{N} Y_{i j} V_{j 0} \cong\left(\mathrm{P}_{\mathrm{i}}^{\mathrm{sp}}-\mathrm{jQ}_{\mathrm{i}}^{\mathrm{sp}}\right) / V_{i 0}^{*}
$$

Substituting (9) into (8) and dividing both sides by $V_{i}$, yield

\footnotetext{
3 - Fast Decoupled Load Flow
}

$\sum_{j=1}^{N} Y_{i j} \Delta V_{j}+\frac{P_{i}^{s p}-j Q_{i}^{s p}}{\left(V_{i 0}^{*}\right)^{2}} \Delta V_{i}^{*} \cong \Delta V_{i}, i=1,2, \ldots, N, i \neq s(10)$

In the above equation, the current mismatch $\Delta I_{i}^{s p}$ is determined by

$$
\begin{aligned}
& \Delta I_{i}^{s p}=\left(\mathrm{P}_{\mathrm{i}}^{\mathrm{sp}}-\mathrm{jQ}_{\mathrm{i}}^{\mathrm{sp}}\right) / V_{i 0}^{*}-\sum_{j=1}^{N} Y_{i j} V_{j 0} \\
& =\frac{\left(\mathrm{P}_{\mathrm{i}}^{\mathrm{sp}}-\mathrm{jQ}_{\mathrm{i}}^{\mathrm{sp}}\right)-\left(\mathrm{P}_{\mathrm{i}}-\mathrm{jQ}_{\mathrm{i}}\right)}{V_{i 0}^{*}}=\frac{\Delta P_{i}-j \Delta Q_{i}}{V_{i}^{*}} \\
& \qquad \Delta P_{i}=P_{i}^{s p}-P_{i} \\
& \text { Where } \Delta Q_{i}=Q_{i}^{s p}-Q_{i}
\end{aligned}
$$

The above equation can be written in a matrix forms as follows:

$$
Y_{B U S} \Delta V+D \Delta V^{*}=\Delta I
$$

Where

$$
\begin{aligned}
& \Delta V=\left[\Delta V_{1}, \Delta V_{2}, \ldots, \Delta V_{N}\right], \\
& \Delta I=\left[\Delta I_{1}, \Delta I_{2}, \ldots, \Delta I_{N}\right], \\
& \mathrm{D}=\operatorname{Diag}\left[\left(\mathrm{P}_{\mathrm{i}}^{\mathrm{sp}}-\mathrm{jQ}_{\mathrm{i}}^{\mathrm{sp}}\right) / \mathrm{V}^{* 2}\right]
\end{aligned}
$$

As a static load model [9] has been used in this study, P$\mathrm{Q}$ buses are specified and an initial voltage $V_{i}$ is assigned to each bus. $V_{i}$ is a complex value, and only $\mathrm{N}$ complex divisions are required in calculating diagonal matrix D. Expanding equation (12), it is shown in [7] that only diagonal matrix $\mathrm{D}$ and current mismatch $\Delta I$ are required to be updated in the iteration procedure. As a result computation time reduces roughly to $40 \%$ and $50 \%$ compared with the FDLF and the $\mathrm{NR}^{4}$ methods respectively. In this case reliable convergence characteristic and precise answer is honorable.

\section{Genetic Algorithm Implementation}

The first step for using GA is coding the search space. In this step a string is assigned for each member of search space.

In this case there is a network with 32 buses and it is assumed three sizes of DG units are available. These sizes are 300,600 and 900KVA.Ten percent of the capacity of each unit is dedicated to reactive power generation. Each bus is assigned with one gene in which, zero means that there is no DG in the bus, 1 resembles a 300KVA DG unit,2 resembles 600KVA DG unit and 3 resembles 900KVA DG unit. Next step is making first population in a random manner. In this step there is an important item to be checked. DG penetration should not exceed $1500 \mathrm{KVA}$ ( $30 \%$ of feeder load in peak hours) .

In the third step, load flow program runs both in normal condition and when there is a voltage sag in eventful buses (buses 9,14,23 and 29). Fitness of each chromosome (potential answers) is determined.

Here are the steps to be followed in the next step:

a. Choosing parents with Roulette Wheel.

b. Implementing cross over operator.

\footnotetext{
4 - Newton-Raphson
} 
c. Implementing mutation operator.

d. Two members of new generation are saved and treated. as parents in the next generation.

e. Best answer goes to the next generation.

$a$ to $e$ procedures should be repeated to generate a limited number of individuals (200 individuals). In each step the condition for not exceeding 1500KVA is checked. Finally the member in the last generation with the best fitness value is the optimal answer to the optimization problem.

\section{Simulation Results}

1) Loss Reduction: In this section GA only calls power loss objective function. The optimization algorithm suggests installing DG units in 9, 15, 16 and 25 buses. It is observed that when loss minimization goal is followed the algorithm suggests the lowest available size of DG units near highly loaded buses to prevent flowing current in long lines.

Below diagram shows a reduction of $63 \%$ in the network loss.

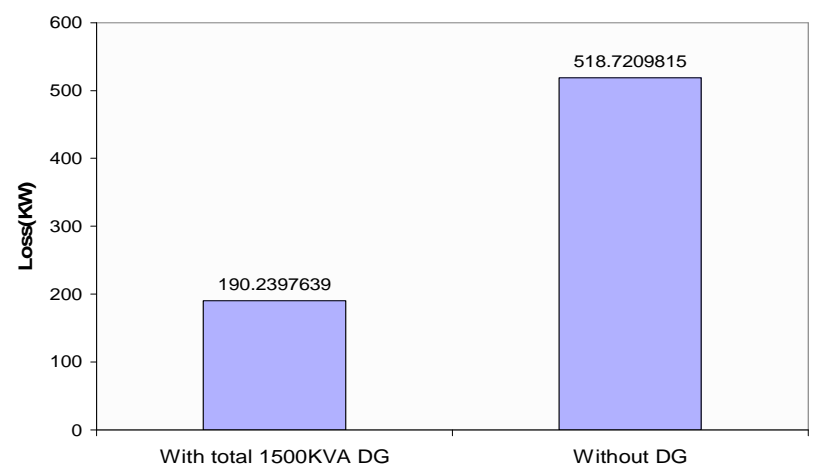

Fig. 2 Loss Reduction in Test Network

2) Voltage Profile Improvement: In this section GA calls the voltage profile improvement fitness function. Proposed algorithm suggests installing a 900KVA DG unit in bus 16 and a 600KVA DG unit in bus19.It is observed that optimization algorithm proposes installing DG units in end feeder buses with poor voltage profile characteristics. Paying attention to the network configuration, it is observed that two main branches are connected to the slack bus (upper main branch, called U branch and lower main branch, called L branch in this case study). Due to this special network configuration installing DG units in each of the two main branches has no effect on the voltage profile of the other main branch. As voltage profile is extremely low in the end feeder buses of $U$ branch, proposed algorithm suggests installing DG units in these buses and the voltage profile in $\mathrm{L}$ branch makes no difference in this case. Figure 3 shows this behavior.

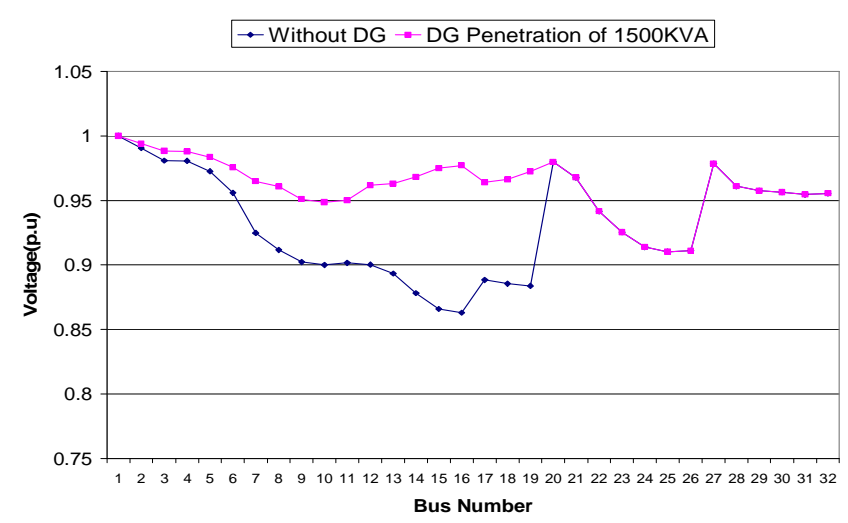

Fig. 3 Voltage Profile Improvement in Test Network

3) Voltage Support in Voltage-Sensitive buses: If only voltage support in sensitive buses and mitigating voltage sags is the goal of optimization problem, genetic algorithm uses objective functions (4) and (5) depending on desired approach. (4) is used when voltage support in voltage sensitive buses is desired and (5) is used when reducing number of buses which experience deep voltage sags is desired. Figures 4 and 5 show voltage support in two sensitive buses of test network (bus 13 and 24) during voltage sag occurrence in eventful buses $(9,12,23$ and 29). Eventful buses have the highest annual fault rate. Hear, desired approach is voltages support in sensitive buses.

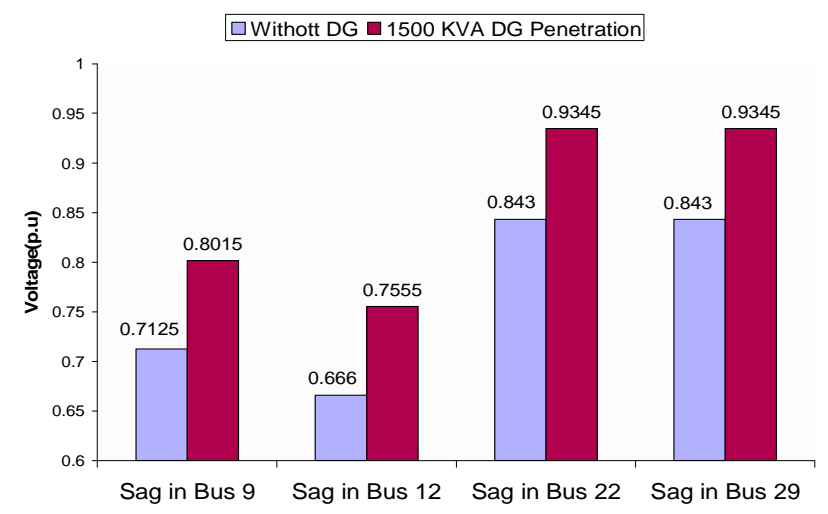

Fig.4. Voltage in Bus 13 during Voltage Sag Occurrence in Eventful Buses

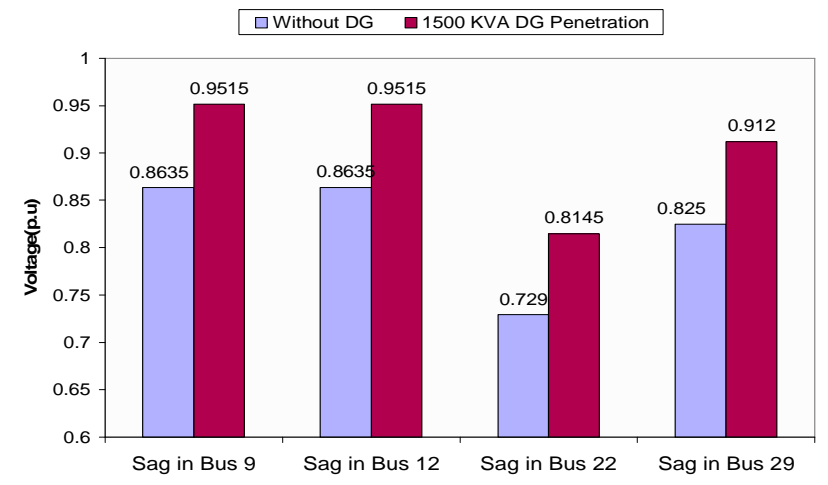

Fig. 5 Voltage in Bus 24 during Voltage Sag Occurrence in Eventful Buses

4) Comprehensive objective function: In this section all three mentioned functions are gathered to form one single objective function which follows all three desired goals. 
After normalizing each of discussed functions, comprehensive objective function is:

$$
f=K_{1} f_{\text {loss }}+K_{2} f_{v}+K_{3} f_{\text {senu }}
$$

In order to magnify the role of each goal, related weighting coefficient $\left(K_{1}, K_{2}\right.$ and $\left.K_{3}\right)$ can become enlarged. For $K_{1}=K_{2}=K_{3}=1$, table I shows proposed location and size of DG units.

TABLE I. Proposed Size and Location For DG Units

\begin{tabular}{|c|c|}
\hline Suggested Bus & DG Size (KVA) \\
\hline 16 & 300 \\
\hline 18 & 600 \\
\hline 25 & 600 \\
\hline
\end{tabular}

Power loss reduces from $518 \mathrm{kw}$ to $193 \mathrm{kw}$. Figure 5 shows voltage profile improvement in the test network.

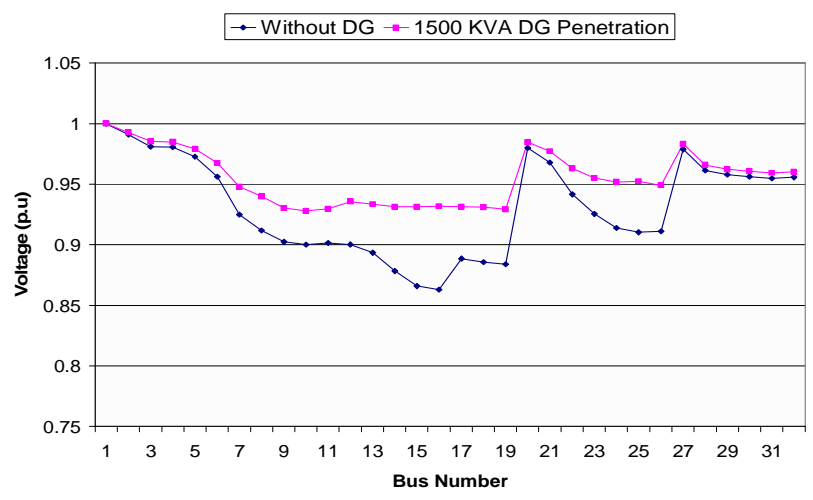

Fig.6 Voltage Profile When Applying Comprehensive Objective Function

Figures 6 and 7 show voltage support in sensitive buses.

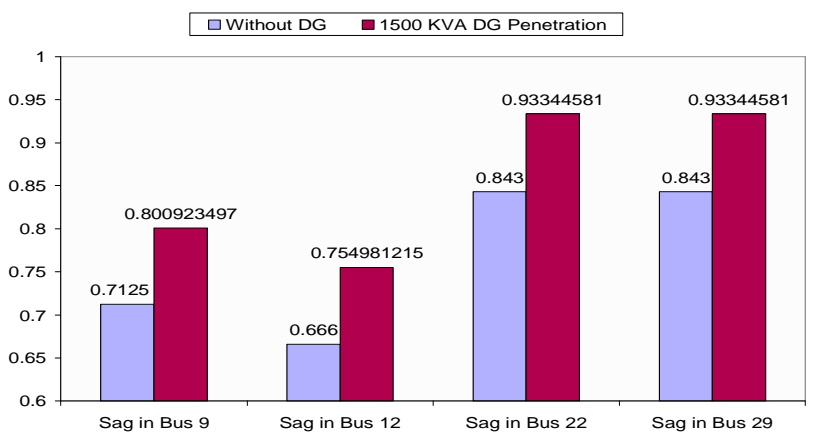

Fig.7 Voltage in Bus 13 When Applying Comprehensive Objective Function

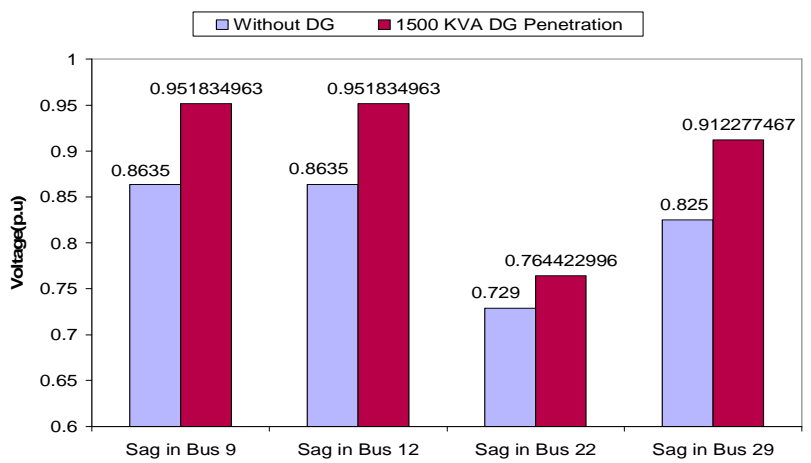

Fig.8. Voltage in Bus 24 When Applying Comprehensive Objective Function
Loss reduction, improvement in voltage profile and voltage support in sensitive buses show the acceptable efficiency of proposed algorithm.

\section{Conclusion}

Due to high employment of voltage sensitive equipments, voltage support in sensitive loads are a great concern for utility companies. Beside network operation cost is directly affected by power loss. In this papaer a long and highly loaded $20 \mathrm{KV}$ feeder has been under investigation to find optimal location and sizing for DG units to support $30 \%$ of the feeder load. This DG penetration level is reasonable due to economic considerations. The presence of two highly voltage sensitive loads in this feeder have made network expansion planners think of voltage sag mitigation and voltage support in these buses. Beside, because of ill-conditioned network, Loss reduction and voltage profile improvement have been of great concern as well. Objective functions with different approaches have been defined and different scenarios were investigated. Results show significant reduction in power loss in addition to voltage profile improvement. Capability of proposed method for voltage sag mitigation in sensitive buses has been also investigated and was proved to be within acceptable limits.

\section{References}

[1] Caisheng.Wang, Hashem Nehrir, "Analytical approaches for optimal placement of distributed generation sources in power system", IEEE Transaction on Power Systems, Vol 19, No 4, November 2004, pp.2068 - 2076

[2] R. Gnativ, J. V. Milanovic, "Voltage Sag Propagation In Systems With Embedded Generation And Induction Motors", Power Engineering Society Summer Meeting, 2001. IEEE, Volume 1, Issue, 2001 pp.474 - 479

[3] Angelo Baggini, Handbook of Power Quality, First Edition, the Atrium, Southern Gate, Chichester, West Sussex PO19 8SQ, England John Wiley \& Sons Ltd, 20

[4] M. J. Jahromi, E. Farjah, M, Zolghadri, "Mitigating Voltage Sag By Optimal Allocation of Distributed Generation Using Genetic Algorithm", This paper appears in: Electrical Power Quality and Utilization, 2007,Barcelona .pp 1-6

[5] D. E. Goldberg, Genetic Algorithms in Search ,Optimization and Machine Learning, Addison Wesley,1989.

[6] S. F.Mekhamer,

Hawary,S.A.Soliman,M.A.Moustafa,M.M.Mansour, "New Heuristic Strategies for Reactive Power Compensation of Radial Distribution Feeders," IEEE trans. Power Delivery,Vol. 17, , October 2002, pp. 1128-1135

[7] Yan Zhang, J.V.Milanovic, "Application of Niching Genetic Algorithms in System-Wide Voltage Sag Mitigation Studies", Power-Tech, 2007.IEEE, pp. 1515-1521

[8] Young-Hyun Moon; Byoung-Kon Choi; Byoung-Hoon Cho; SeHo Kim; Bok-Nam Ha; Jung-Ho Lee, "Fast and reliable distribution system load flow algorithm based on the $\mathrm{Y}_{\mathrm{Bus}}$ formulation", Power Engineering Society Summer Meeting, 1999. IEEE

Volume 1, Issue, 18-22 Jul 1999, pp. 238 - 242

[9] T. Gozel, M. H. Hocaoglu, A. Baliki, "Optimal Placement and Sizing of Distributed Generation on Radial Feeder with Different Static Load Models", International Conference on Future Power Systems, 2005. IEEE, pp. 2-6 